\title{
Percepciones de los estudiantes sobre el uso de los laboratorios virtuales en mecánica de suelos
}

\author{
Julio Esteban Colmenares ${ }^{a}$, Norma Rocio Héndez ${ }^{b} \&$ Jorge Celis-Giraldo ${ }^{b}$ \\ ${ }^{a}$ Facultad de Ingeniería, Universidad Nacional de Colombia, Bogotá D.C., Colombia. jecolmenaresm@unal.edu.co \\ ${ }^{b}$ Grupo de Investigación STEM+B, Universidad Nacional de Colombia, Bogotá D.C., Colombia.nrhendezp@unal.edu.co, jecelisg@unal.edu.co
}

\begin{abstract}
Resumen - Este artículo muestra los resultados de adoptar laboratorios virtuales en la asignatura de mecánica de suelos en los pregrados de ingeniería civil e ingeniería agrícola en la Universidad Nacional de Colombia. Desde la perspectiva de los estudiantes, presenta de qué manera los laboratorios contribuyen a la comprensión y aplicación de conceptos en la solución de problemas relacionados con la mecánica de suelos. Se exploraron los resultados de la percepción de autoeficacia de los estudiantes así como de su percepción sobre los laboratorios virtuales en relación con las características sociodemográficas de los estudiantes. La evidencia recolectada sugiere que los estudiantes encuentran que los laboratorios contribuyen a potenciar sus habilidades de investigación, de análisis y conocimientos en mecánica de suelos. Los estudiantes que dedicaron mayor tiempo a la asignatura consideran que los laboratorios virtuales permiten desarrollar conceptos y habilidad de análisis y evalúan positivamente el trabajo con estos laboratorios.
\end{abstract}

Palabras clave - Laboratorios virtuales; mecánica de suelos; formación en ingeniería; aprendizaje basado en problemas

Recibido para revisar Octubre 22 de 2017, aceptado Noviembre 16 de 2017, versión final Noviembre 7 de 2017

\section{Perceptions of students about the use of virtual laboratories in soil mechanics}

Abstract - This article presents the results of using virtual laboratories in the soil mechanics course. The course is part of the Civil and the Agricultural engineering curriculums at the Universidad Nacional de Colombia. From the students' point of view, the use of virtual laboratories contributes to the understanding and application of concepts in order to solve problems related to soil mechanics. The results of students' perception of self-efficacy are explored as well as their perception of virtual laboratories in relation to the sociodemographic characteristics of students. Evidence collected suggests that students find laboratories useful to improve their research and analytical skills and help to expand their knowledge about soil mechanics. High performance students, in the course, consider that the use of virtual laboratories is key to acquire engineering concepts and analytical skills. They assess as positive the use of virtual laboratories.

Keywords - Virtual laboratories; soil mechanics; engineering education; problem-based learning.

\section{Introducción}

Desarrollar competencias para la comprensión y aplicación de conceptos en la solución de problemas es fundamental en la formación de ingenieros [1,2]. Para los estudiantes de pregrado que cursan la asignatura mecánica de suelos, el origen y formación de suelos, estados de esfuerzos totales y efectivos, incrementos de esfuerzos, conductividad hidráulica, resistencia al corte y compresibilidad son conceptos [3-8] a aprender en los laboratorios [9,10]. Investigaciones sobre laboratorios virtuales señalan que éstos contribuyen a desarrollar competencias [1114]. Sin embargo la revisión de trabajos sobre el uso de dichos laboratorios en mecánica de suelos evidencia que existe poca experiencia y la mayoría de aplicaciones provienen de países desarrollados [15-17]. Este artículo describe las percepciones de los estudiantes sobre la utilidad de los laboratorios virtuales para comprender y aplicar conceptos en la solución de problemas relacionados con mecánica de suelos. Para tal fin, se adoptaron unos laboratorios virtuales en el segundo semestre del año 2016 en la asignatura mecánica de suelos en los pregrados de ingeniería civil e ingeniería agrícola en la Universidad Nacional de Colombia.

La asignatura mecánica de suelos hace parte del área de geotecnia. Se orienta al desarrollo del perfil del egresado en ingeniería civil que incorpora una serie de objetivos educativos, dos de los cuales están relacionados con el propósito de los laboratorios virtuales que aquí se presentan: "Articular sus conocimientos técnicos y científicos para la resolución congruente con el contexto social, cultural, económico, político y medio ambiental, de problemas de ámbito nacional o internacional en conjunto con varias disciplinas" y "Tener una inclinación especial hacia el aprendizaje, la profundización y la investigación continua y permanente para ser capaz de asimilar y adaptarse rápidamente a los cambios (ej.: científicos, tecnológicos) a los que se enfrente en su vida profesional" [18]. La asignatura tiene un peso en el plan curricular de 3 créditos académicos ${ }^{1}$ [19]. Durante las 16 semanas que comprende el semestre, los estudiantes dedican unas 144 horas de las cuales el $44,4 \%$ corresponde a trabajo presencial que incluye el desarrollo de clases teóricas, talleres con ejercicios sobre la teoría estudiada y laboratorios presenciales [20]. En los laboratorios presenciales se realizan ensayos estandarizados cuyo conocimiento se considera indispensable para la

Como citar este artículo: Colmenares, J.E., Héndez, N.R. and Celis-Giraldo, J., Percepciones de los estudiantes sobre el uso de los laboratorios virtuales en mecánica de suelos. Educación en Ingeniería, 13(25), pp. 88-101, Febrero, 2018.

${ }^{1}$ Según el Acuerdo 033 de 2007 del Consejo Superior Universitario, un crédito académico equivale a 48 horas de trabajo académico, que incluyen la dedicación presencial y el trabajo independiente del estudiante. 
formación en el campo de la geotecnia y debido al plan de estudios y al tiempo disponible, se aborda un número limitado de ensayos. Los ensayos que se priorizan son consolidación, compresión inconfinada, compresión triaxial, corte directo y conductividad hidráulica. De los cinco ensayos de laboratorio mencionados, consolidación y corte directo se realizaron en modalidad virtual y presencial, mientras que los tres restantes se hicieron únicamente bajo modalidad presencial.

Es importante anotar que el $73 \%$ de los profesores encuestados en el último proceso de autoevaluación del programa de ingeniería civil considera que los laboratorios contribuyen a la calidad del programa, especialmente a la comprensión de los conceptos mientras el 37\% de los estudiantes entre 5\% y $10 \%$ coinciden con esta afirmación [21]. De otro lado, el 23\% de los profesores afirma que se requiere mejorar la infraestructura de los laboratorios y el 57\% de los estudiantes presenta observaciones frente a la suficiencia de equipos y materiales [21]. El programa de ingeniería civil cuenta con infraestructura para realizar 36 tipos de prácticas de suelos, rocas y pavimentos, temáticas del componente de geotecnia, un promedio de 10 equipos por práctica y una capacidad de atención simultánea de 30 estudiantes [21]. El mantenimiento de estas máquinas y el desarrollo de ensayos de extensa duración, más de un día de ejecución, generan altos costos [22] y dificultan la participación de todos los estudiantes en la realización de todos los laboratorios en modalidad presencial. Ante este panorama, en el año 2016 se propuso el diseño de los laboratorios virtuales con el fin de aportar a los objetivos de la asignatura mecánica de suelos. Por ello, el diseño consideró superar las dificultades de logística e impactar en las habilidades asociadas con la resolución de problemas $[2,23]$. En este proceso, los objetivos de la asignatura fueron asociados con tres tipos de logro de aprendizaje: 1) la comprensión y conocimiento de conceptos 2) las habilidades analíticas y 3) las habilidades en investigación desde la perspectiva del aprendizaje basado en problemas [11].

\section{Marco conceptual}

Los laboratorios virtuales se diseñaron desde la perspectiva del aprendizaje basado en problemas (ABP) con el fin de aportar a la capacidad de resolver problemas relacionados con la mecánica de suelos [24]. El análisis inicial acerca de los laboratorios permitió identificar que éstos se consideran como una herramienta para el aprendizaje y el desarrollo de habilidades, pero que debido a la manera en que son implementados no alcanzan los resultados esperados [20]. En este contexto, se asumió el aprender como una acción orientada a la comprensión de conceptos [25] que acontece en un sistema social [26] en el cual el conocimiento está distribuido entre los individuos y sus ambientes, incluyendo objetos, artefactos, herramientas y las comunidades de las cuales hacen parte [27] Diseñar un laboratorio desde la perspectiva del ABP supuso plantear una situación que involucrara un problema relacionado con las propiedades mecánicas de los suelos, a partir del cual los estudiantes iniciaran una ruta de análisis y una solución que involucrara el uso de ensayos y datos de laboratorio. Un problema se entendió como una situación que enfrenta un estudiante o un grupo de ellos y requiere una solución que no es evidente al principio [28]. Solucionar un problema depende del conocimiento y los conceptos que tuvieran los estudiantes [1,29], y también de sus habilidades de investigación tales como las de observación, identificación de preguntas o necesidades y planteamiento de estrategias para comprender los fenómenos. En el proceso de solución de los problemas, los estudiantes definen un problema, determinan estrategias y recursos para encontrar soluciones e identifican el tipo y la fuente de datos requeridos [20]. Con estos insumos, seleccionan opciones de solución [28] y pueden evaluarlas frente a otras para saber cuál es la mejor [30]. Así enfocados, los laboratorios promueven el desarrollo de habilidades de pensamiento y de conceptualización, la aplicación de más modelos y teorías sobre los fenómenos y el fortalecimiento de la autonomía en el proceso de adquisición de habilidades [31].

Un laboratorio "brinda una oportunidad para integrar aspectos conceptuales, procedimentales y epistemológicos dentro de enfoques alternativos, que pueden permitir el aprendizaje de los estudiantes con una visión constructivista a través de métodos que implican la resolución de problemas, los cuales le brindan la experiencia de involucrarse con los procesos de la ciencia” [30, p.103]. Además, la actividad experimental desarrolla una fuerte conexión entre la realidad y la teoría por medio de la observación de conceptos [31]. Los laboratorios pueden desarrollarse de manera presencial, remota o virtual. La manera presencial implica compartir el espacio físico en cual se encuentran los recursos mientras que la remota supone manipular los recursos del laboratorio presencial a través de un software que permita su acceso. La virtual es llevada a cabo a través de simulaciones de los recursos del espacio presencial sin que sea necesario hacer uso de este [32], de manera que una práctica de laboratorio virtual es un "proceso de simulación que extrae del medio real la esencia de un fenómeno determinado" [22, p.77]. Existen diferentes resultados a partir de la implementación de laboratorios virtuales. Según [17], la interacción con las simulaciones aumentó la motivación a estudiar los contenidos de la asignatura. [33] reporta que los laboratorios virtuales incrementaron la motivación, pero no los niveles de aprendizaje. Por su parte, [34] hace alusión no sólo a la modalidad sino a la forma en que se desarrollan los laboratorios, indicando que hay laboratorios catalogados como presenciales pero que son realizados únicamente por el profesor mientras el estudiante es un observador, con lo que la interacción del estudiante con los recursos difiere de lo esperado en un laboratorio presencial. En la investigación de [13] se encontró que los estudiantes tuvieron preferencia por los presenciales sobre los remotos y virtuales, y de los remotos sobre los virtuales. El meta-estudio de [11] no encontró evidencias significativas ni una tendencia clara que permita generalizar la afirmación de que la modalidad presencial sea mejor que la virtual o viceversa. Al respecto [25] menciona que la modalidad a adoptar depende del objetivo que se persigue, aunque siempre será preferible aprovechar ambas modalidades.

En la Tabla 1, se encuentran las competencias a desarrollar asociadas con las categorías de logro educativo que están en estrecha relación con la comprensión de conceptos y solución de problemas en el marco de la asignatura de mecánica de suelos. 
Tabla 1

Relación de las competencias de la asignatura con las categorías de [11]

\begin{tabular}{|c|c|c|c|}
\hline Competencias & $\begin{array}{l}\text { Conocimientos } \\
\text { y comprensión }\end{array}$ & $\begin{array}{c}\text { Habilidades } \\
\text { de } \\
\text { investigación }\end{array}$ & $\begin{array}{c}\text { Habilidades } \\
\text { analíticas }\end{array}$ \\
\hline $\begin{array}{l}\text { Identificar en } \\
\text { situaciones concretas } \\
\text { problemas asociados } \\
\text { con las propiedades } \\
\text { mecánicas de los } \\
\text { suelos }\end{array}$ & $\mathrm{X}$ & $\mathrm{X}$ & \\
\hline $\begin{array}{l}\text { Aplicar técnicas y } \\
\text { herramientas con el } \\
\text { propósito de analizar } \\
\text { las propiedades } \\
\text { mecánicas de los } \\
\text { suelos }\end{array}$ & $\mathrm{X}$ & $\mathrm{X}$ & \\
\hline $\begin{array}{l}\text { Recolectar, } \\
\text { interpretar y analizar } \\
\text { datos que permitan } \\
\text { formular soluciones } \\
\text { que involucran las } \\
\text { propiedades } \\
\text { mecánicas de los } \\
\text { suelos }\end{array}$ & $\mathrm{X}$ & $\mathrm{X}$ & $\mathrm{X}$ \\
\hline
\end{tabular}

Tabla 2

Conocimientos, habilidades y temas para problemas asociados con los diferentes ensayos

\begin{tabular}{|c|c|c|}
\hline $\begin{array}{l}\text { Conocimientos y } \\
\text { comprensión CC }\end{array}$ & $\begin{array}{l}\text { Habilidades de } \\
\text { investigación HI }\end{array}$ & $\begin{array}{c}\text { Habilidades de análisis } \\
\text { HA }\end{array}$ \\
\hline $\begin{array}{l}\text { - Flujo en medios } \\
\text { porosos. } \\
\text { - Comportamiento } \\
\text { volumétrico. } \\
\text { - Comportamiento } \\
\text { de resistencia. }\end{array}$ & $\begin{array}{l}\text { - Identificar cuáles } \\
\text { conceptos son } \\
\text { relevantes para la } \\
\text { solución de un } \\
\text { problema. } \\
\text { - Identificar las } \\
\text { variables que } \\
\text { afectan un } \\
\text { fenómeno o } \\
\text { problema de } \\
\text { ingeniería. } \\
\text { - Proponer arreglos } \\
\text { experimentales } \\
\text { para medir las } \\
\text { propiedades del } \\
\text { suelo necesarias } \\
\text { para aplicar la } \\
\text { teoría en relación } \\
\text { con un problema } \\
\text { dado. } \\
\text { - Identificar las } \\
\text { fuentes de error en } \\
\text { la ejecución de } \\
\text { experimentos. } \\
\text { - Identificar el tipo } \\
\text { de ensayo } \\
\text { apropiado según el } \\
\text { tipo de suelo y las } \\
\text { condiciones del } \\
\text { problema a } \\
\text { estudiar. } \\
\text { - Presentar de } \\
\text { manera organizada } \\
\text { el estudio de un } \\
\text { problema. } \\
\end{array}$ & $\begin{array}{l}\text { - Identificar la influencia } \\
\text { de las condiciones de } \\
\text { realización del ensayo } \\
\text { en los resultados. } \\
\text { - Incorporar análisis de } \\
\text { sensibilidad (estudios } \\
\text { paramétricos) ante } \\
\text { cambios de propiedades } \\
\text { o tipos de suelos, para } \\
\text { comprender mejor el } \\
\text { problema o fenómeno. } \\
\text { - Simplificar y formular } \\
\text { un modelo que en } \\
\text { condiciones } \\
\text { controladas reproduzca } \\
\text { de manera razonable el } \\
\text { fenómeno en estudio, } \\
\text { en relación con un } \\
\text { problema de ingeniería. } \\
\text { - Comparar los } \\
\text { resultados los } \\
\text { experimentales con la } \\
\text { teoría y con resultados } \\
\text { de otros experimentos. } \\
\text { - Elaborar gráficos de } \\
\text { tendencia con los } \\
\text { resultados ex } \\
\text { experimentales. } \\
\text { Elaborar conclusiones } \\
\text { fundamentadas, a partir } \\
\text { de la observación o de } \\
\text { la teoría. } \\
\text { resultados obtenidos. } \\
\text { rentificar }\end{array}$ \\
\hline
\end{tabular}

Fuente: Los autores
Con el fin de seleccionar los laboratorios virtuales se realizó una búsqueda de herramientas y recursos de libre acceso encontrando escasez de laboratorios basados en simulaciones en mecánica de suelos con las cuales sea posible interactuar. En cambio, se encontraron recursos multimedia (videos y animaciones) y guías de laboratorios digitalizadas catalogadas como laboratorios virtuales. Fue indispensable entonces, diseñar nuevos laboratorios virtuales. La virtualidad, en sentido de "virtualidad informática" [35], consistió en la digitalización de una serie de datos producidos en laboratorios reales con los cuales los estudiantes realizaron simulaciones sobre el comportamiento de los suelos partiendo de información real para responder preguntas a partir de hipótesis explicativas que se esperaba comprobar [36]. Por ello, se partía de una situación real para la realización de los laboratorios, luego de una pregunta problema, posteriormente la identificación de aspectos conceptuales, el manejo y finalmente el análisis de datos según las características de cada caso. Los laboratorios tuvieron como propósito el fortalecimiento de tres dimensiones del aprendizaje: conocimientos y comprensión (CC), habilidades de investigación (HI) y habilidades de análisis (HA). Para su diseño fue necesario operacionalizar lo esperado para cada dimensión (Tabla 2), pues se requería establecer los problemas para cada laboratorio.

Además de la definición de problemas, el diseño de los laboratorios implicó la construcción de una guía de laboratorio que incluyó la situación problema y una lista de preguntas. En el primer laboratorio se hizo entrega de una base de datos de resultados de ensayos de caracterización de suelos y consolidación con la finalidad de que los estudiantes realizaran los cálculos correspondientes, analizaran las propiedades de las muestras ensayadas e identificaran la variabilidad de las propiedades de comprensibilidad de los suelos en profundidad. En el segundo laboratorio, a partir de un modelo teórico, acompañado con una base de datos, los estudiantes debían predecir el comportamiento de diferentes muestras de suelo de dos ensayos de laboratorio: el ensayo de compresión inconfinada y el ensayo de compresión triaxial consolidado no drenado.

\section{Evaluación de logros educativos esperados}

La evaluación de los laboratorios virtuales se hizo empleando informes de laboratorio que incluyeron el reconocimiento del problema de suelos presente en la situación, el planteamiento de la estrategia de solución o análisis, el reconocimiento de los conceptos necesarios para comprenderlo, el uso que se daría a los datos recolectados a través del laboratorio, además del análisis o propuesta de solución del problema. El esquema de la V de Gowin [30,37] se consideró adecuado para sinterizar dichos elementos de manera articulada y evidenciando así si el estudiante comprendió el problema, relacionó sus conocimientos y solucionó el problema. De esta manera, se tuvieron en cuenta el dominio conceptual (teorías, principios y conceptos), la recolección y el procesamiento de datos y la presentación de resultados.

\section{Metodología}

La incorporación y evaluación de las tecnologías de información y comunicaciones en escenarios educativos involucra 
Tabla 3

Categorías de análisis

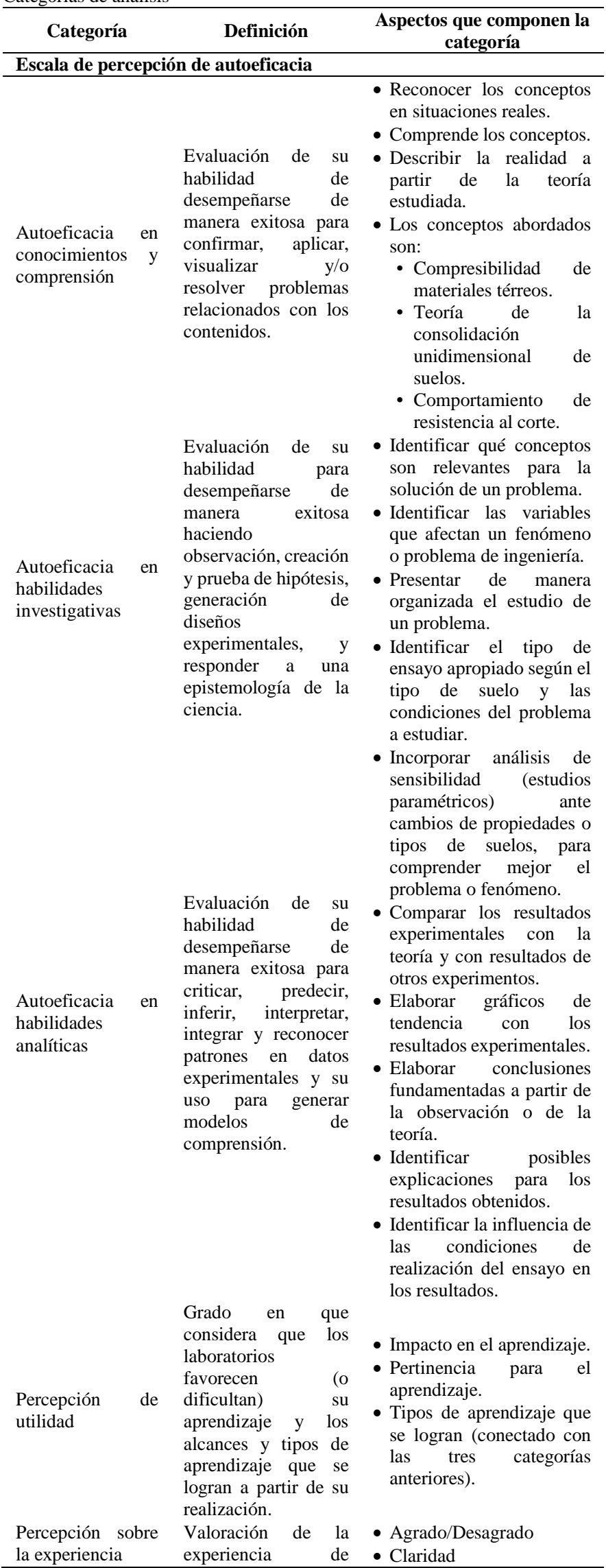

\begin{tabular}{llrll}
\hline Categoría & \multicolumn{2}{c}{ Definición } & \multicolumn{1}{c}{$\begin{array}{c}\text { Aspectos que componen la } \\
\text { categoría }\end{array}$} \\
\hline & realización de los & $\bullet$ Facilidad/Dificultad & \\
& $\begin{array}{l}\text { laboratorios } \\
\text { términos positivos y }\end{array}$ & $\begin{array}{l}\text { ・ Necesidad } \\
\text { acompañamiento }\end{array}$ & de \\
& $\begin{array}{l}\text { negativos para los } \\
\text { estudiantes. }\end{array}$ & & \\
\hline
\end{tabular}

Fuente: Los autores

diferentes dominios de las instituciones como políticas, recursos, planes de estudios, organización, prácticas de enseñanza, prácticas de aprendizaje [38], y contextos como el político, administrativo, de aula, prácticas y estudiantes [39]. Este estudio se centró particularmente en el contexto del aula así como de las prácticas de enseñanza y aprendizaje [40]. Algunos estudios acerca de laboratorios virtuales en educación se han orientado a evaluar los conocimientos adquiridos $[13,14,41]$, su utilidad frente al aprendizaje $[13,17,41]$, o aspectos técnicos como su usabilidad y funcionamiento [17,41]. En este estudio, el interés estuvo centrado en la comprensión de conceptos y el desarrollo de habilidades para resolver problemas relacionados con las propiedades mecánicas de los suelos a través de tres dimensiones asociadas con dicha habilidad que, como se presentó en el marco conceptual, hacen parte de los posibles aprendizajes derivados de la implementación de laboratorios: CC, HI y HA [11]. Se evaluó la percepción de autoeficacia como indicador de aprendizaje en dichas categorías, así como la percepción asociada con la utilidad y con la evaluación de la experiencia.

\subsection{Categorías de análisis}

Para el análisis de la información recolectada se definieron cinco categorías a partir de la revisión bibliográfica realizada y los propósitos del estudio (Tabla 3).

Además, en el proceso de análisis de los datos surgieron subcategorías y la categoría emergente "experiencias previas con laboratorios". Esta alude a las realizaciones previas con laboratorios y las valoraciones acerca de ellos, que como se evidencia en los resultados influye significativamente en la percepción sobre la experiencia con estos laboratorios.

\subsection{Recolección de información}

\subsubsection{Instrumento 1. Cuestionario}

El cuestionario aplicado al inicio del segundo semestre de 2016 tuvo como objetivos: 1) caracterizar socio-demográfica y académicamente a los estudiantes que cursaron la asignatura 2) identificar la percepción de los participantes acerca de sus conocimientos y habilidades (de investigación y análisis) asociadas con la resolución de problemas en el momento de iniciar el curso y 3) identificar la percepción que tienen los estudiantes sobre el papel que juegan los laboratorios, presenciales y virtuales, en su aprendizaje de acuerdo con sus experiencias previas. El cuestionario tenía tres secciones. La primera con preguntas de caracterización socio-demográfica. La segunda con preguntas construidas a partir de la escala de percepción de auto-eficacia planteada por [42] para quien la 


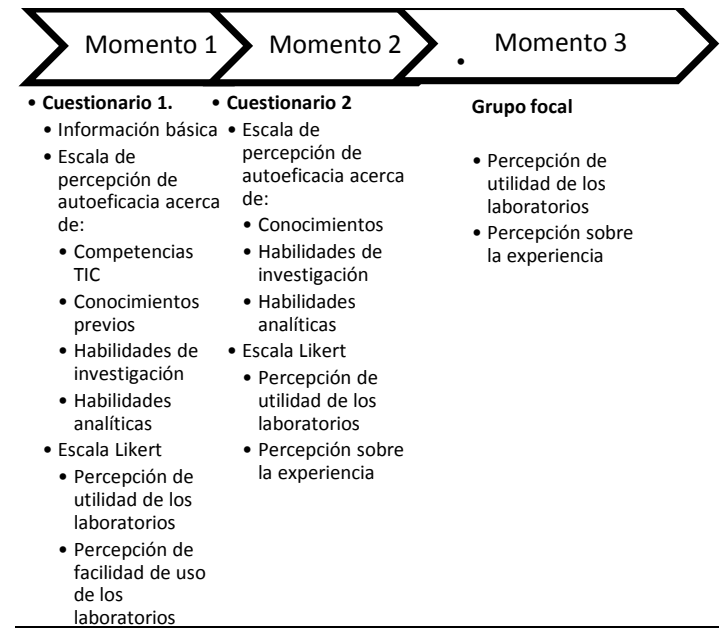

Figura 1. Momentos de la investigación e instrumentos Fuente: Los autores

percepción de auto-eficacia se trata de las creencias de los individuos sobre sus habilidades para producir determinados logros y se relaciona con la percepción de dificultad que se debe superar para lograr un resultado, en este sentido, la auto-eficacia está relacionada con una capacidad y no con una actitud, intención o disposición. Según [42], la percepción de autoeficacia influye en que los individuos piensen de manera errática o estratégica, de manera optimista o pesimista frente a determinadas situaciones, lo que establece una posible relación entre esta autopercepción y los niveles de desempeño, tema que no se evalúa en este estudio pero puede ser pertinente de revisar en otros. La tercera incluyó preguntas con una escala Likert de 4 opciones de respuesta, donde 1 correspondía a totalmente en desacuerdo y 4 a totalmente de acuerdo. Esta escala permite medir la intensidad de los sentimientos hacia un aspecto propuesto [43], en este estudio la experiencia con los laboratorios. Este instrumento fue validado por medio de una rúbrica, ítem por ítem, a través de tres pares expertos en mecánica de suelos. Los criterios de evaluación fueron univocidad (el ítem evalúa un solo aspecto), pertinencia (el ítem tiene relación lógica con la pregunta de estudio) e importancia (el ítem es relevante en relación con la pregunta de estudio). La validación fue complementada con la realización de un piloto. El instrumento se ajustó según los resultados y su versión final fue aplicada de manera presencial en la tercera semana académica a los 30 estudiantes que iniciaron la asignatura. En ese encuentro los estudiantes completaron un formato de consentimiento informado que contenía los propósitos de la investigación y la autorización para usar la información recogida durante el semestre, con este y con otros instrumentos $\mathrm{y}$ actividades.

\subsubsection{Instrumento 2. Cuestionario}

El segundo instrumento tuvo como objetivos: 1) caracterizar socio-demográfica y académicamente a los estudiantes. Esta información se solicitó nuevamente dado que ambos cuestionarios eran anónimos y solicitarla cada vez era indispensable para establecer relaciones entre la información socio-demográfica y las demás respuestas 2) identificar la percepción de los participantes acerca de sus CC, HI y HA determinadas como logro de los laboratorios implementados 3) identificar la percepción de utilidad de los laboratorios virtuales realizados para el aprendizaje, así como el tipo de logros que estos permiten alcanzar 4) describir las percepciones sobre la experiencia de desarrollo de los laboratorios realizados. La estructura fue similar a la del primer cuestionario. En la primera sección se añadieron preguntas de trayectoria académica y dedicación como el número de asignaturas y los créditos cursados durante el semestre. En la segunda sección las preguntas de auto-percepción de eficacia que incluyeron las tres dimensiones de interés del estudio. Los ítems fueron encaminados hacia los logros que se esperaba fortalecer con los laboratorios virtuales implementados. Finalmente, la sección con preguntas tipo Likert incluyó ítems para evaluar la experiencia de desarrollo de los laboratorios implementados. De esta manera, aunque los dos instrumentos tenían estructuras similares, las preguntas fueron diferentes por lo que no se trató de la aplicación de un ejercicio de evaluación pre y post en términos de comparabilidad, sino de una valoración en dos momentos del proceso de escolarización: previo a la implementación de los laboratorios virtuales y posterior a la experiencia de realización de los mismos. El segundo instrumento se validó por medio de tres pares expertos, dos de ellos especialmente en mecánica de suelos y otro en el campo de la educación. Las dimensiones de evaluación fueron como en el primer instrumento: univocidad, pertinencia e importancia. Igualmente, tuvieron la posibilidad de comentar cada ítem y el instrumento en su conjunto. Recibidas sus observaciones, se ajustó para obtener la versión final la cual se dispuso en la herramienta Forms de Google ${ }^{\circ}$, para distribuirlo vía correo electrónico. El instrumento fue aplicado en la última semana de la asignatura. Para este momento, el grupo contaba con 27 estudiantes en total, de los cuales 22 dieron respuesta.

\subsubsection{Instrumento 3. Grupo focal}

El grupo focal se propuso como técnica de recolección de información complementaria a los cuestionarios. Con este se buscó profundizar en las percepciones de los estudiantes sobre los laboratorios virtuales implementados y su relación con el aprendizaje y el desarrollo de habilidades para la resolución de problemas. Los objetivos del grupo focal fueron: 1) identificar la percepción que tienen los estudiantes sobre los laboratorios como estrategia didáctica en ingeniería 2) conocer la percepción sobre la experiencia de desarrollo de los laboratorios virtuales implementados en el marco de esta investigación en la asignatura mecánica de suelos y 3) identificar las percepciones de los estudiantes sobre los laboratorios virtuales como herramienta para el aprendizaje y el desarrollo de la habilidad para resolver problemas en la asignatura mecánica de suelos. La selección de participantes se realizó buscando representación de estudiantes con buen desempeño académico, regular y bajo, según las calificaciones obtenidas a lo largo del semestre. Se contó con la participación de 9 estudiantes en total. Al inicio del grupo focal, se solicitó completar un formato de consentimiento informado y autorización del uso de la información allí recolectada. 


\begin{tabular}{|c|c|c|c|c|}
\hline Nombre & Fundam & tado & Densid & \\
\hline 2: Acompañamiento & 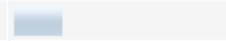 & 6 & i & 1 \\
\hline AE Conocimientos & 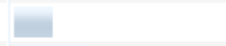 & 5 & - & 1 \\
\hline 2 AE Habilidades de análisis & 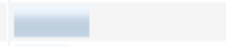 & 9 & 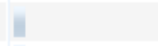 & 1 \\
\hline 2 AE Habilidades investgación & 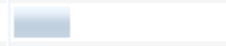 & 7 & I & 1 \\
\hline Datos & E & 4 & 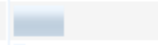 & 3 \\
\hline Dedicación & i & 2 & I & 1 \\
\hline Definición de laboratorio & + & 8 & 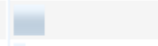 & 2 \\
\hline 淊 Desagrado & 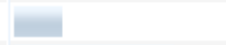 & 6 & - & 1 \\
\hline Dinámica de desarrollo & 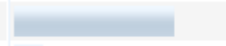 & 18 & 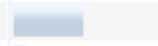 & 4 \\
\hline 2*: Evaluación & vat & 4 & i & 1 \\
\hline Percepción sobre la experiencia & 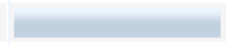 & 23 & 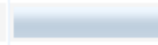 & 8 \\
\hline 湝 Experiencias de laboratorios & 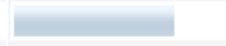 & 18 & 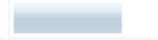 & 6 \\
\hline Falta de agencia & & 1 & i & 1 \\
\hline 2: Guias - instrucciones & 1 & 2 & $\square$ & 2 \\
\hline 没 Habilidades prácicas & E & 4 & I & 1 \\
\hline Percepción de uflidad & & 0 & 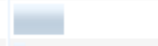 & 3 \\
\hline Perinencia & & 1 & I & 1 \\
\hline Retroalimentación & E & 3 & I & 1 \\
\hline 2: Tipo de aprendizaje & 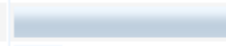 & 26 & 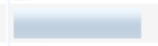 & 7 \\
\hline 2 Utilidad & 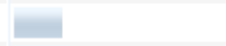 & 6 & $\square$ & 2 \\
\hline 2 Vinculo asignatura - lab & 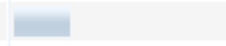 & 7 & 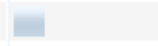 & 2 \\
\hline
\end{tabular}

Figura 2. Frecuencia de categorías

Fuente: Los autores por medio del programa de análisis de datos cualitativos Atlas ti.

\subsubsection{Tratamiento de la información recolectada}

La información recolectada de los cuestionarios recibió tratamiento estadístico a partir del cual se identificaron las medias frente a cada pregunta y cada categoría. De igual manera, se establecieron relaciones con aspectos de la caracterización socio-demográfica como el género, el promedio académico y el tiempo dedicado a la asignatura durante el semestre. Por su parte, la información derivada del grupo focal fue transcrita en su totalidad y posteriormente sujeta a análisis y categorización usando para ello el programa Atlas.ti. En la Fig. 2 se presenta la frecuencia con que una subcategoría o categoría fue aplicada, es decir, la cantidad de citas que se le atribuyeron (columna Fundamentado). Es pertinente indicar que algunas citas se ubicaron en varias categorías por su contenido. En la figura también se muestra la cantidad de vínculos que cada subcategoría o categoría tiene con otras (columna Densidad).

En la Fig. 3 se presenta la red de subcategorías y categorías que se construyó a partir del análisis en la cual se evidencia la densidad indicada en la Fig. 2.

\section{Resultados}

\subsection{Caracterización socio-demográfica y trayectoria académica}

Las caracterizaciones, tanto socio-demográfica como de trayectoria académica, que se presentan recogen los datos de los 22 estudiantes que respondieron el cuestionario, del total de 27 que concluyeron la asignatura. La distribución por género fue 90,91\% hombres y 9,09\% de mujeres. El porcentaje de mujeres

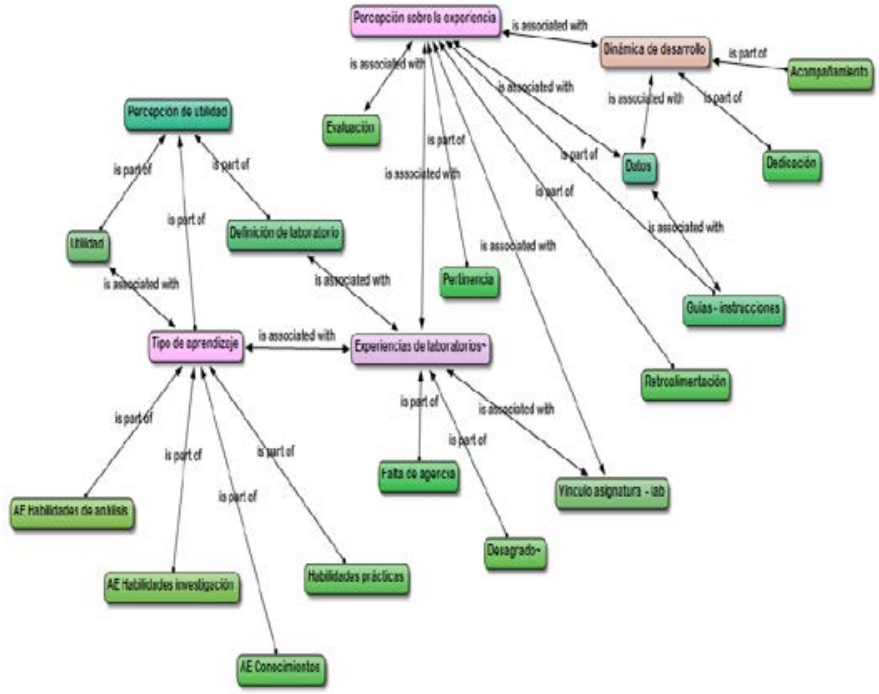

Figura 3. Red de relaciones entre categorías

Fuente: Los autores por medio del programa de análisis de datos cualitativos Atlas ti.

es menor al de mujeres matriculadas en la Universidad Nacional de Colombia en los programas de ingeniería agrícola e ingeniería civil, que para 2015 fue de 29,46\% y 17,93\%, respectivamente [44]. Respecto al rango de edad, el 13,64\% era menor de 20 años y el 4,55\% mayor de 30. La mayor concentración de estudiantes estaba en el rango entre los 20 y 24 años (68,18\%). Acerca del origen, el 59,09\% eran de Bogotá y el 40,91\% de otras 8 ciudades del país. En relación con el tipo de institución educativa en la que cursaron la educación media, el 54,55\% provenía de instituciones públicas. El 54,55\% de los estudiantes desempeñó algún trabajo durante el semestre en que se implementaron los laboratorios virtuales. De los 22 estudiantes, el 90,91\% estaban matriculados en ingeniería civil y el 4,55\% en ingeniería agrícola. El 13,64\% de los estudiantes provenía de un Programa de Admisión Especial. En cuanto a la repitencia en la asignatura mecánica de suelos, el 36,36\% de los estudiantes se encontraba en esta condición. El promedio del "promedio aritmético ponderado acumulado" (PAPA) de los estudiantes era 3,76 siendo 3 el menor promedio presentado y 4,7 el mayor. En relación con el número total de créditos académicos cursados, el promedio era de 96,23 créditos cursados siendo 50 el mínimo de créditos cursados y 129 el máximo. En promedio los estudiantes tenían matriculados 17,64 créditos académicos.

\subsection{Percepción de autoeficacia}

La percepción de autoeficacia de cada dimensión (CC, HI y HA) se evaluó a través de una serie de ítems frente a los cuales cada estudiante debía calificar de 0 a 100 . De esta manera se obtuvo un resultado por cada ítem para el grupo de estudiantes y de cada estudiante por cada categoría que engloba los diferentes aspectos. La categoría CC se evaluó a través de 20 ítems relacionados con los tres conceptos centrales que se buscaba fortalecer con la implementación de los laboratorios virtuales: flujo en medios porosos, comportamiento y el máximo 1.925. Haciendo una escala que divide 


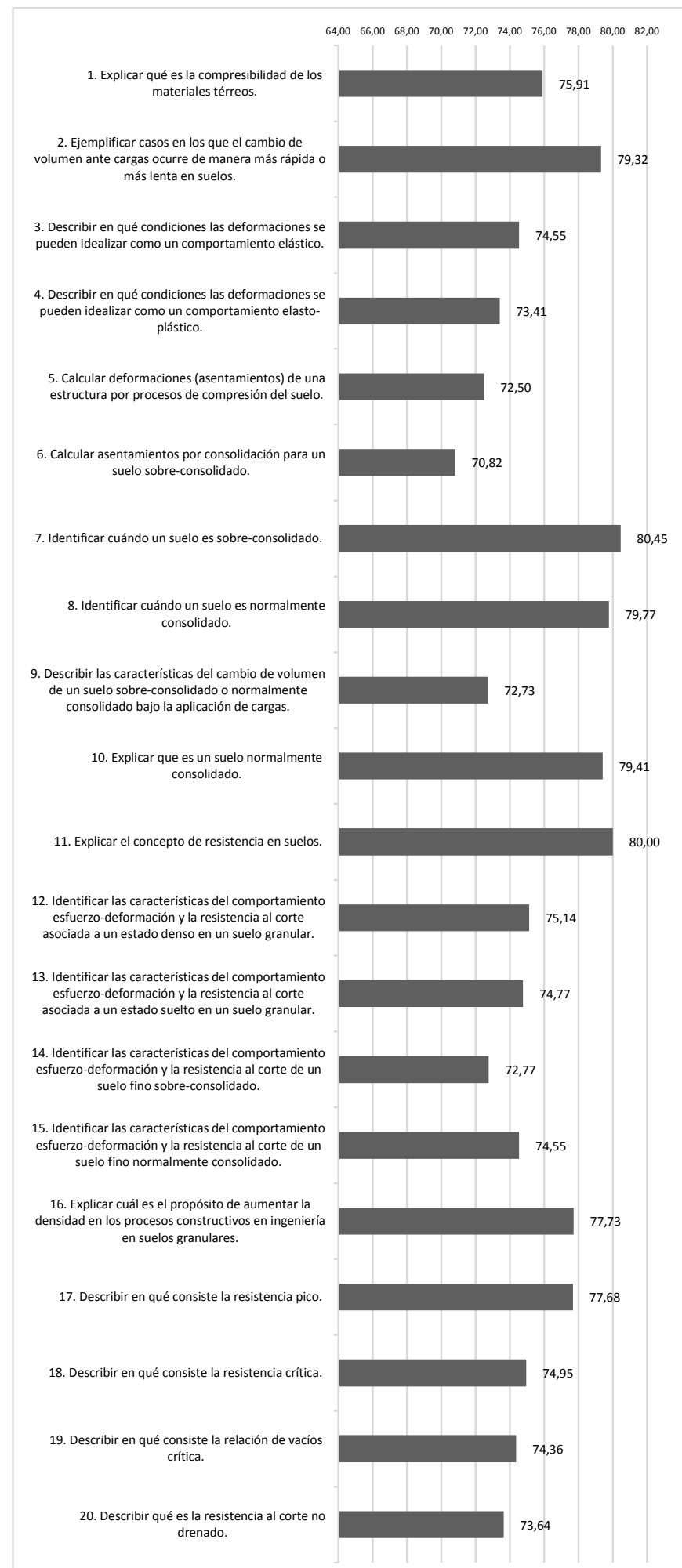

Figura 4. Puntaje promedio de ítems de la categoría de CC Fuente: Los autores

volumétrico y comportamiento de resistencia. La autopercepción de eficacia de cada estudiante en esta categoría podría puntuar máximo 2.000 puntos. El promedio de puntaje fue de 1.514 puntos siendo el puntaje mínimo registrado 1.340 el rango posible de puntaje en cuatro (mínimo, bajo, alto y máximo), este puntaje promedio se encuentra clasificado como máximo. Los ítems con mayor promedio en la calificación fueron identificar cuándo un suelo es sobre consolidado $(80,45)$ y explicar el concepto de resistencia de suelos (80). Los de menor promedio fueron calcular asentamientos por consolidación para suelos sobre-consolidados $(70,81)$ y deformaciones (asentamientos) de una estructura por procesos de compresión del suelo (72,5) (Fig. 4).

Para evaluar la percepción de autoeficacia de HI se construyeron 23 ítems. La categoría por estudiante podía recibir una calificación máxima de 2.300 puntos. El puntaje promedio fue $1.684,45$ siendo 2.010 el mayor puntaje y 1.290 el menor. Haciendo una escala que divide el rango posible de puntaje en cuatro (mínimo, bajo, alto y máximo), este puntaje promedio se encuentra clasificado como alto. Los ítems con mayor puntaje promedio son los relacionados con identificar qué tipo de datos producto de ensayos de laboratorio se requieren para proponer soluciones ante un problema de suelos $(79,09)$, cuáles son los conceptos relevantes ante un problema de mecánica de suelos $(77,86)$ y las implicaciones de que el cambio de volumen ante cargas ocurra de manera lenta en un suelo (77,32) (Fig. 5). Mientras que con menor puntaje promedio se encuentran determinar, según la información que se tenga del perfil del suelo, si la información que se puede obtener con un ensayo de consolidación es pertinente para solución un problema dado (65), y definir, según las características de un problema de suelos, cuándo es pertinente realizar un ensayo de resistencia al corte $(68,82)$.

Por su parte, para evaluar la percepción de autoeficacia de las HA se aplicaron 23 ítems. El puntaje máximo para esta categoría correspondía a 2.300. El puntaje promedio de esta dimensión fue 1652,82 siendo el puntaje mayor 2150 y el menor 800 , muy por debajo del promedio. Haciendo una escala que divide el rango posible de puntaje en cuatro (mínimo, bajo, alto y máximo), este puntaje promedio se encuentra clasificado como alto. Los ítems de mejor puntaje promedio fueron explicar las causas por las cuales los ensayos no consolidados - no drenados alcanzan la misma resistencia en términos de esfuerzos totales independientemente de la presión de confinamiento (65) e identificar el tipo de ensayo de resistencia a realizar, el cual en condiciones controladas debe reproducir de manera razonable el fenómeno en estudio, en relación con un problema de ingeniería $(65,36)$ (Fig. 6). Mientras que con mayor puntaje están construir gráficos que presenten los resultados de un ensayo realizado $(77,95)$ y contrastar los resultados obtenidos en diferentes ensayos $(77,18)$.

\subsection{Percepción de utilidad de los laboratorios como estrategia didáctica y para el aprendizaje}

Las percepciones fueron identificadas a través de ítems que fueron calificados a través de una escala Likert de cuatro opciones. Se construyeron 12 ítems para evaluar la percepción de utilidad de los laboratorios virtuales, tres más para evidenciar el tipo de logro que se puede alcanzar a través de dichos laboratorios y otros tres enfocados a lo que se requiere para su desarrollo. Para el primer grupo de preguntas el puntaje máximo 


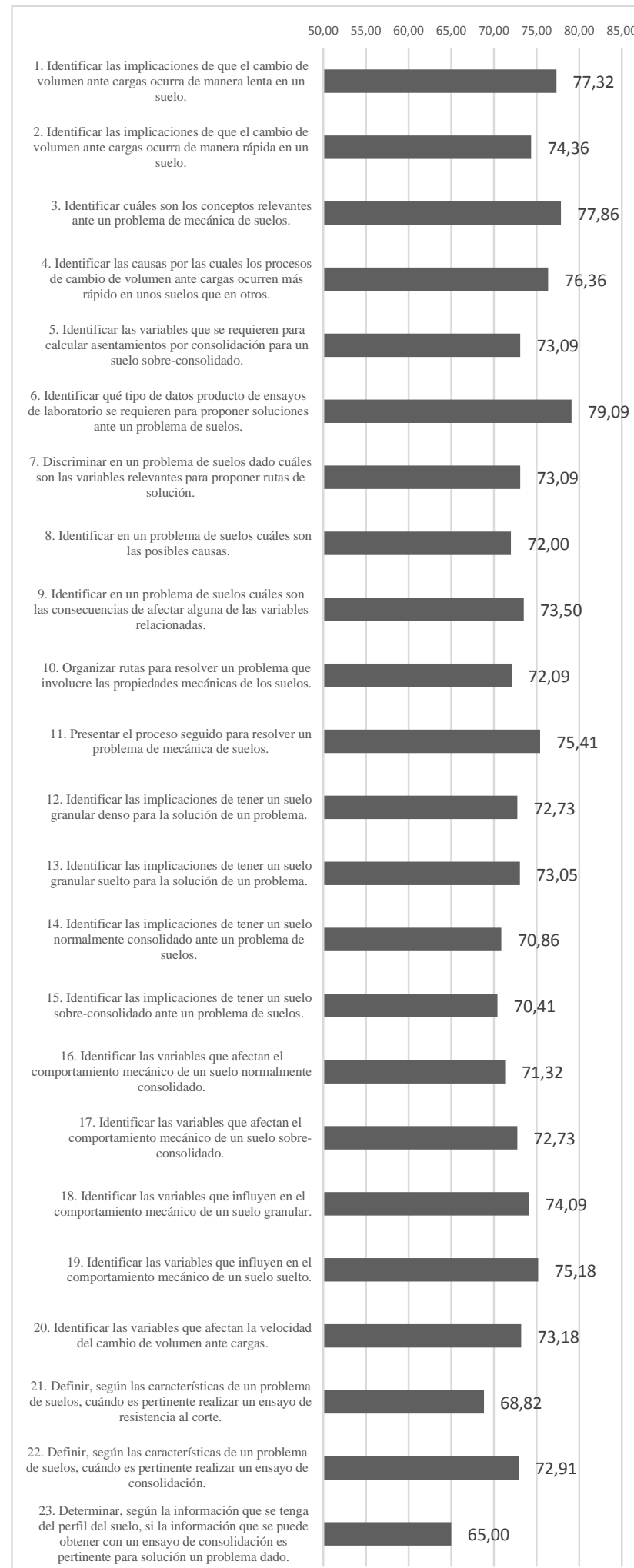

Figura 5. Puntaje promedio de ítems de la categoría de HI Fuente: Los autores

posible es 48 , siendo 4 el máximo puntaje por pregunta. El puntaje promedio fue 33,95 , siendo 13 el menor puntaje obtenido y 41 el mayor. Los ítems frente a los que se expresó en promedio mayor grado de acuerdo fueron "El desarrollo de este tipo de actividades es útil para aprender a solucionar problemas de ingeniería.” (3,19); "El desarrollo de las actividades de laboratorios virtuales en la asignatura me permite poner en práctica conceptos y teorías.” $(3,09)$; y, “El desarrollo de estas actividades me prepara para mi rol como profesional en ingeniería.” $(3,09)$. Por su parte, la afirmación frente a la que en promedio hubo menor acuerdo fue "Quisiera que se incluyan más actividades como estas en la asignatura.” (2,55) (Fig. 7).

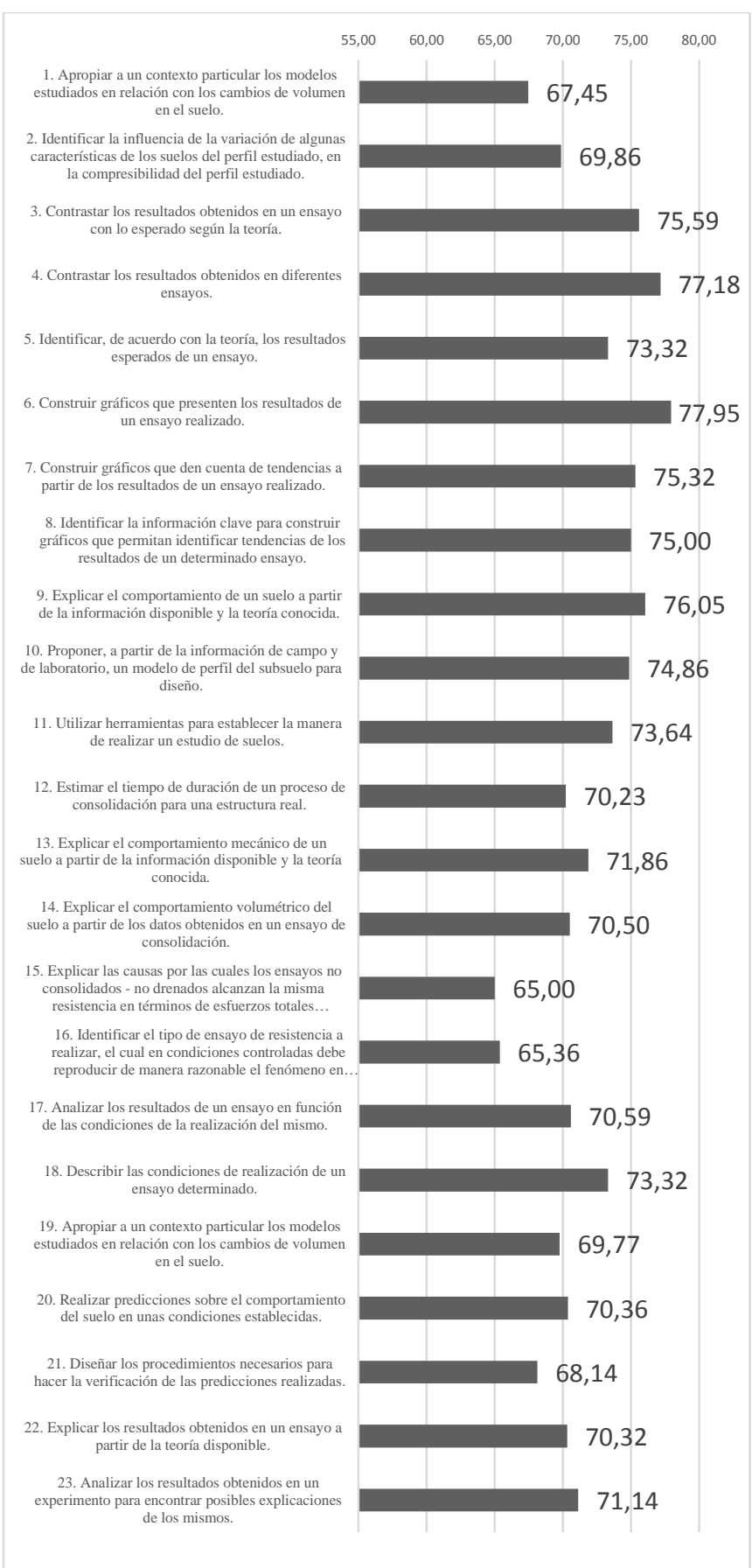

Figura 6. Puntaje promedio de ítems de la categoría de HA. Fuente: Los autores 


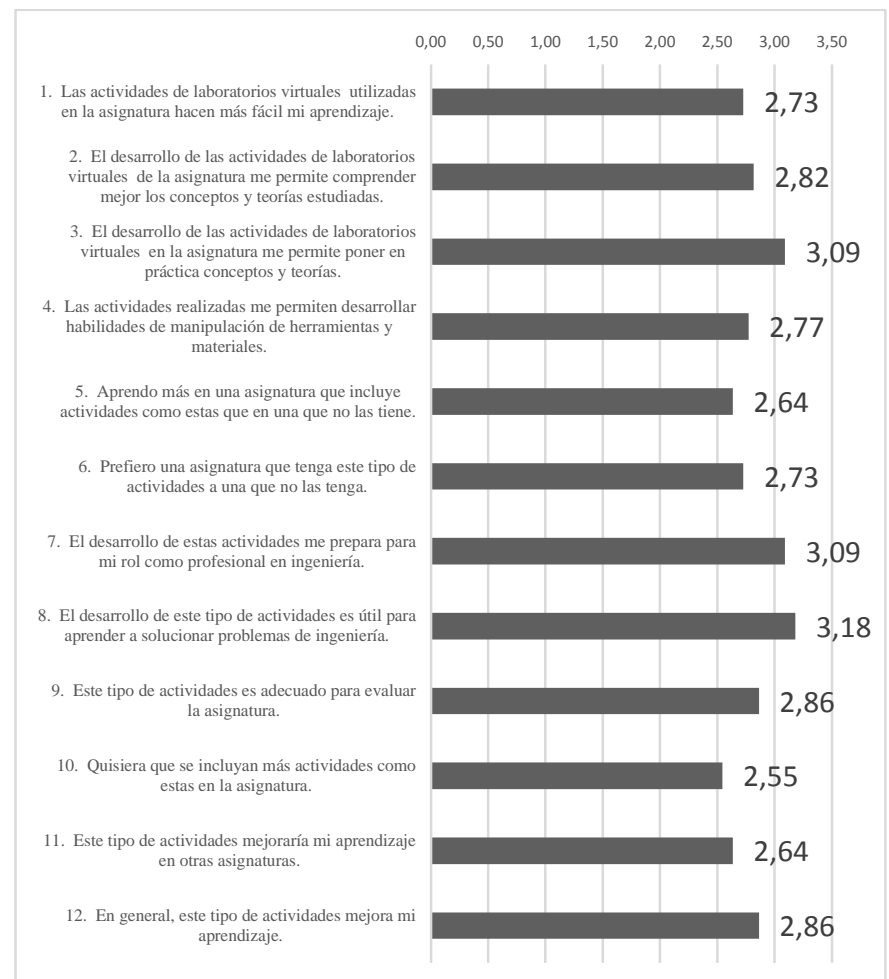

Figura 7. Puntaje promedio de ítems de la categoría de percepción de utilidad Fuente: Los autores

De acuerdo con los estudiantes, los laboratorios permiten interactuar con casos cercanos a la realidad, se constituyen en escenarios de paso de la teoría a la práctica y permiten poner a prueba modelos estudiados. Estas concepciones son relevantes para la percepción sobre la utilidad que tienen de estos como estrategia didáctica. De otro lado, los estudiantes expresaron en el grupo focal que su participación en la ejecución de los ensayos de laboratorio realizados a lo largo de la carrera ha sido limitada en tanto estos son ejecutados por los laboratoristas frente a un grupo de estudiantes a quienes luego se les hace entrega de los datos obtenidos. Los estudiantes en ocasiones no participan en el proceso completo, ni toman decisiones sobre la ejecución. Dadas las características de los ensayos, la organización de los laboratorios y los tiempos disponibles los estudiantes no están teniendo participación directa en los laboratorios presenciales. Un análisis de las concepciones sobre los laboratorios que tienen los estudiantes junto con los comentarios realizados acerca de su ejecución, sugiere una ruptura entre la expectativa y la experiencia; perciben que los laboratorios tienen un potencial didáctico subutilizado. Además, según su percepción, la falta de participación en la ejecución de los ensayos de laboratorio disminuye su comprensión y dificulta el análisis de los datos. En consecuencia, aunque se encontraron percepciones positivas sobre los laboratorios como herramienta didáctica, algunos estudiantes no consideran relevante que se utilicen en otras asignaturas ni que generen mayor aprendizaje. En el segundo grupo de afirmaciones, orientadas a identificar el tipo de alcance de aprendizaje que se puede lograr con el desarrollo de los laboratorios, la afirmación con la que en promedio hubo

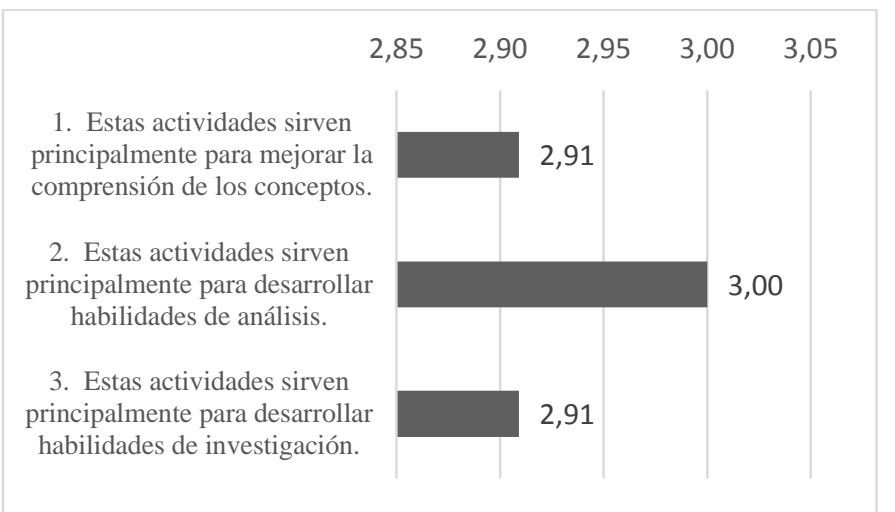

Figura 8. Puntaje promedio de ítems de la categoría de percepción de utilidad, alcance

Fuente: Los autores

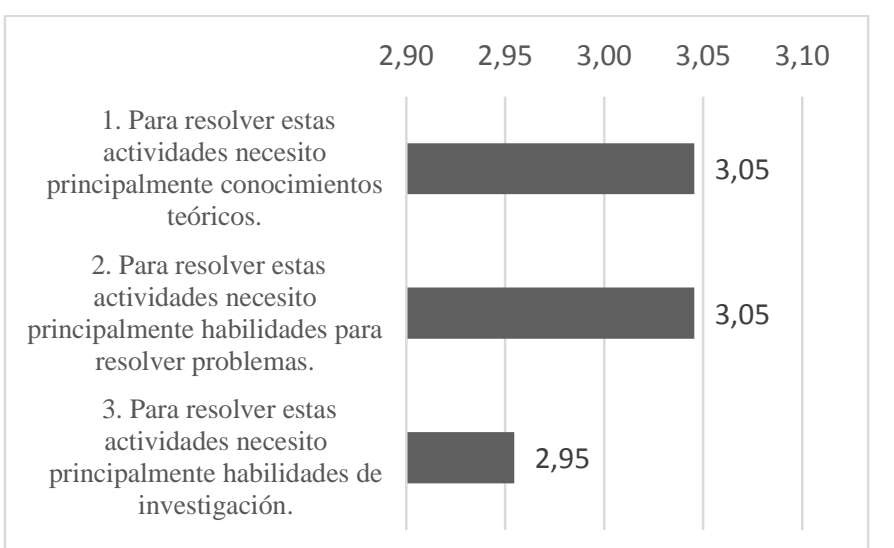

Figura 9. Puntaje promedio de ítems de la categoría de percepción de utilidad, requerimientos

Fuente: Los autores

mayor acuerdo fue "Estas actividades sirven principalmente para desarrollar habilidades de análisis.” (3) (Fig. 8). Por su parte, en el tercer grupo, afirmaciones orientadas a identificar las necesidades frente al desarrollo de los laboratorios, las afirmaciones con las que en promedio hubo mayor acuerdo fueron "Para resolver estas actividades necesito principalmente conocimientos teóricos" y "Para resolver estas actividades necesito principalmente habilidades para resolver problemas", ambas con un puntaje promedio de 3,05 (Fig. 9).

En el grupo focal se hizo referencia a habilidades prácticas y competencias generales como el trabajo en equipo. Se encontró que para los estudiantes existe una articulación profunda entre la realización del ensayo y el análisis de los datos que este produce. $\mathrm{Al}$ respecto perciben que la realización de laboratorios pierde utilidad por la falta de contexto suficiente en relación con el origen de los datos, situación que dificulta su comprensión de los mismos. Por otra parte, el trabajo en equipo y el uso de herramientas como Excel para producir las gráficas y analizar los datos fueron otros logros de aprendizaje mencionados por los estudiantes, los cuales pertenecen al rango de competencias generales. Algunos aprendizajes asociados con habilidades de análisis que se destacan son: cuestionarse el origen y fiabilidad de los datos, analizar las causas de los resultados obtenidos, organizar 


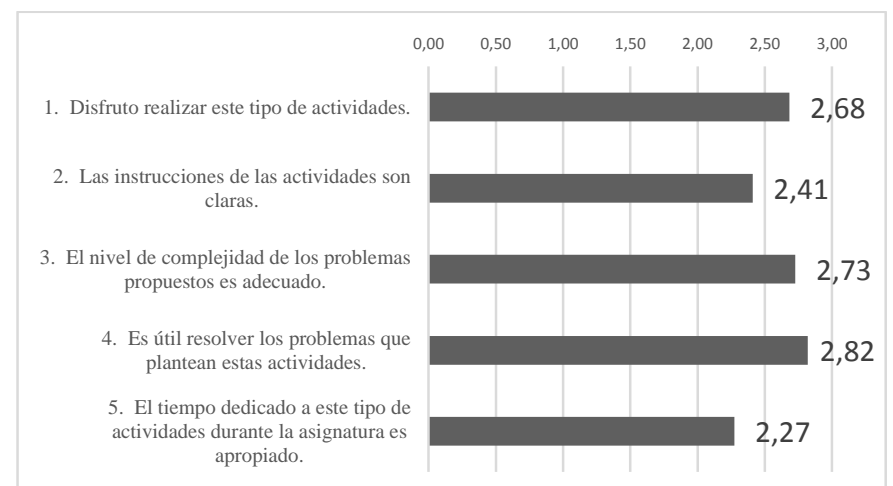

Figura 10. Puntaje promedio de ítems de la categoría de evaluación de la experiencia, aspectos generales

Fuente: Los autores

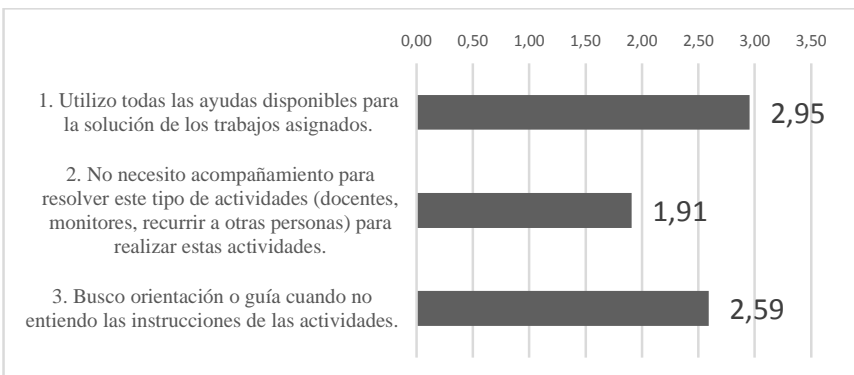

Figura 11. Puntaje promedio de ítems de la categoría de evaluación de la experiencia

Fuente: Los autores

los datos recibidos en función de las preguntas problema, tomar decisiones acerca de cuáles son los datos necesarios y qué hacer con ellos. Así, aunque percibieron como abrumadora la cantidad de datos recibidos, encontraron estrategias para comprenderlos, trabajarlos y orientarse hacia la solución de las situaciones planteadas.

\subsection{Evaluación de la experiencia}

En esta categoría se incluyeron 8 afirmaciones que fueron calificadas por los estudiantes a través de una escala Likert de 4 opciones. De este grupo, 5 afirmaciones evaluaban de manera general la experiencia y 3 la necesidad de ayuda y solicitud de la misma por parte de los estudiantes. En cuanto a la evaluación general de la experiencia de desarrollo de los laboratorios virtuales, la afirmación que en promedio generó mayor acuerdo fue "Es útil resolver los problemas que plantean estas actividades.” con puntaje de 2,82. Mientras que la que generó mayor desacuerdo fue "El tiempo dedicado a este tipo de actividades durante la asignatura es apropiado.”, con un puntaje promedio de 2,27 (Fig. 10).

$\mathrm{Al}$ indagar sobre el acompañamiento en el desarrollo de los laboratorios, el mayor descuerdo se dio frente a la afirmación "No necesito acompañamiento para resolver este tipo de actividades (docentes, monitores, recurrir a otras personas)" con un puntaje promedio de 1,91. Por su parte, la afirmación que mayor acuerdo encontró fue "Utilizo todas las ayudas disponibles para la solución de los trabajos asignados.” con un puntaje promedio de 2,95 (Fig. 11).

En el grupo focal, entre los aprendizajes destacados asociados con la investigación está buscar información teórica que permita comprender los datos disponibles y el problema en sí mismo, probar diferentes rutas y tomar decisiones sobre cuáles son las más pertinentes. Sobre los conocimientos y su comprensión, los estudiantes consideraron relevante la oportunidad de poner la teoría en función de los laboratorios, apropiar los conceptos y abstraer de la teoría, aquellos conocimientos requeridos para analizar un problema. Un elemento crítico reiterado por los estudiantes fue la incertidumbre frente a los datos recibidos, lo que suponía relacionar la situación, las preguntas y los datos. Aunque desde la percepción de algunos, ésto generó dificultades y desmotivación, desde la óptica del diseño de la investigación este desequilibrio hace parte de la transformación de los laboratorios desde el ABP.

Los estudiantes se refirieron a la necesidad de acompañamiento inicialmente para la comprensión de la tarea y la organización de la ruta de trabajo y posteriormente, frente a las dudas del proceso y los resultados obtenidos. Durante el desarrollo de la asignatura, los estudiantes contaban con sesiones de asesoría con el docente, la disponibilidad de un monitor y la posibilidad de consultar con los laboratoristas. En el segundo laboratorio se desarrolló una sesión especial de acompañamiento para resolver inquietudes. Para los estudiantes fue insuficiente el acompañamiento que brindaron los laboratoristas frente a sus dudas. Al respecto, los estudiantes no percibieron suficientes las herramientas y personas de acompañamiento en el proceso de desarrollo de la actividad, mientras que el equipo docente no percibió alta demanda de solicitudes, dado que en el segundo laboratorio se hizo una sesión específica de acompañamiento en la que no hubo gran participación de los estudiantes. Adicionalmente, los estudiantes se refirieron a la importancia de la retroalimentación como forma de evaluar sus decisiones en el proceso y para afianzar sus estrategias de abordaje de los datos y el análisis de los mismos. Según los estudiantes, recibieron muchos datos que no venían organizados de manera que pudieran abordarlos fácilmente, por lo que la primera etapa de desarrollo fue extenuante y descontó tiempo para el análisis. Al respecto los estudiantes consideraron que para futuros laboratorios, es indispensable su participación en el ejercicio de toma de datos o que el formato en que se entreguen les permita conocer mayor información sobre el desarrollo del ensayo. A pesar de las dificultades, los estudiantes consideran valioso el cambio de dinámica que se propuso a través de los laboratorios virtuales para retar su capacidad de aplicar conocimientos a situaciones específicas, para experimentar su rol como ingenieros y para leer y comprender un problema determinado.

\section{Análisis de resultados}

En esta sección se presenta el análisis de los resultados presentados previamente como percepción de autoeficacia y percepción de utilidad según las características sociodemográficas y de trayectoria académica de los estudiantes. 


\subsection{Percepción de autoeficacia según el tipo de estudiantes}

Las mujeres puntúan más alto en su percepción de autoeficacia en las tres dimensiones -CC, HA y HI-, de la misma manera que quienes cursaron más créditos durante el semestre y quienes reportaron una menor dedicación de tiempo semanal para la asignatura. Los estudiantes que hacen parte de programas de admisión especial tienen mayor percepción de autoeficacia en CC y HI. (Figs. 12, 13 y 14).

\subsection{Percepción de utilidad como estrategia didáctica según tipo de estudiante}

Las afirmaciones acerca de la utilidad de los laboratorios virtuales como estrategia de aprendizaje fueron calificadas por los estudiantes, en escala de 1 a 4 (1: totalmente en desacuerdo y 4: totalmente de acuerdo) en promedio con 2,83. Al profundizar el análisis de estos resultados en relación con las características socio-demográficas y de trayectoria académica se encontró que los estudiantes con menores PAPA, los que menos tiempo dedicaron al desarrollo de los laboratorios, aquellos que tuvieron actividad laboral durante el semestre y quienes reportaron dedicar más horas a la asignatura presentaron mayor acuerdo con estas afirmaciones. Las sentencias con las que fue reiterado el acuerdo aluden a que el desarrollo de estas actividades los prepara para su rol como ingenieros, es útil para aprender a solucionar problemas y que permite poner en práctica conceptos y teorías. En este mismo sentido, la experiencia de desarrollo de estos laboratorios virtuales les permite experimentar su rol como futuros ingenieros e interpela a sus conocimientos y a su capacidad de ponerlos en práctica en el abordaje de una determinada problemática de mecánica de suelos. De otro lado, quienes presentaron más desacuerdo con las afirmaciones fueron los estudiantes con mejores PAPA y quienes cursaron mayor cantidad de créditos durante el semestre (Fig. 15). El mayor desacuerdo estuvo en relación con que desearían que haya más actividades como estas en otras asignaturas y que se aprende más en una asignatura en la que se desarrollan este tipo de actividades que en una en la que no las hay.

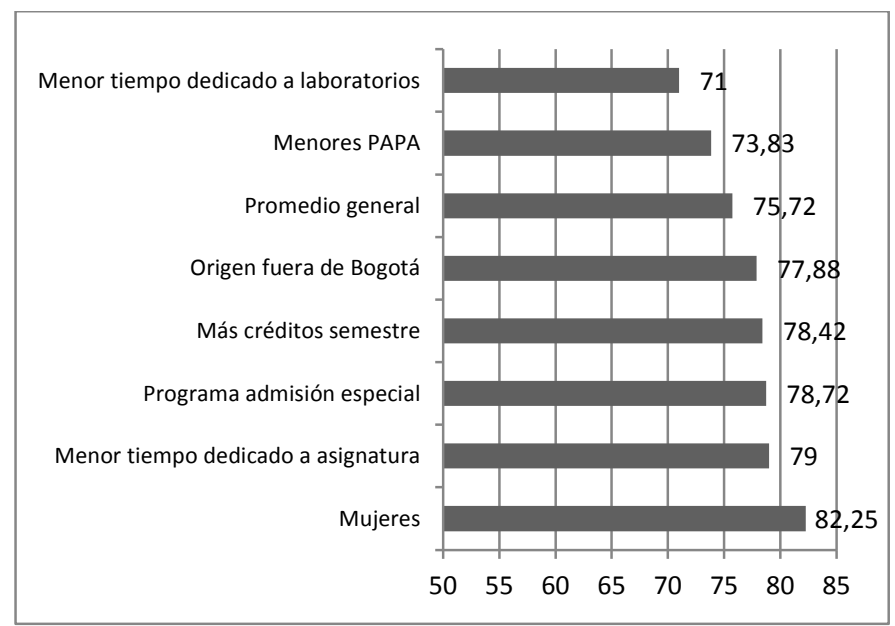

Figura 12. Percepción de autoeficacia en CC según aspectos socio demográficos y de trayectoria académica

Fuente: Los autores

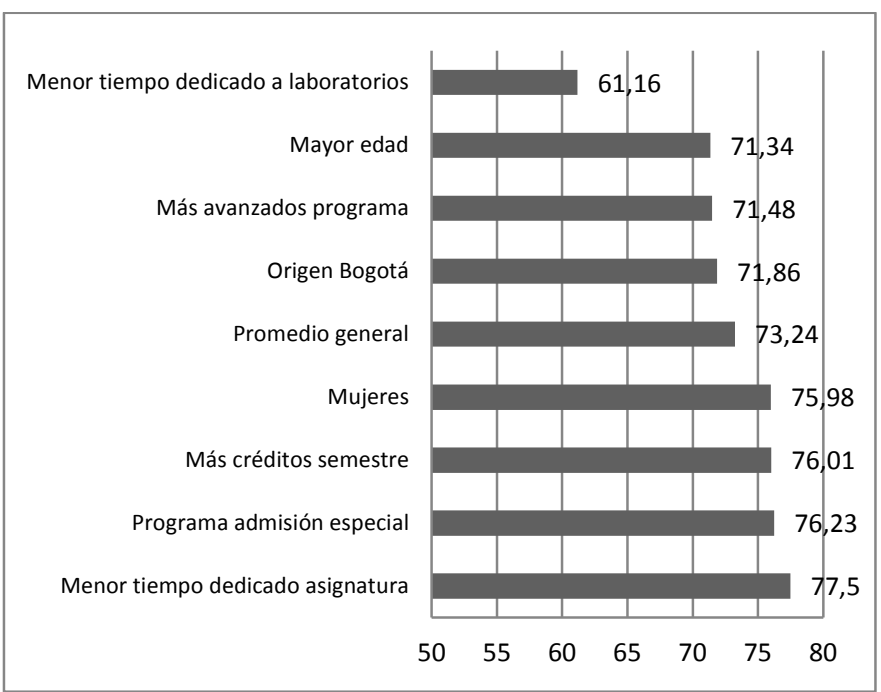

Figura 13. Percepción de autoeficacia en habilidades de investigación (HI) según aspectos socio-demográficos y de trayectoria académica Fuente: Los autores

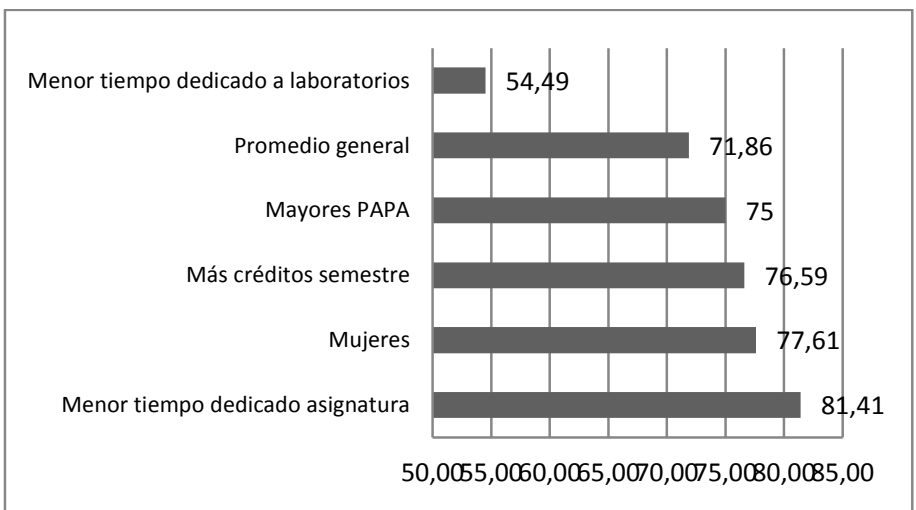

Figura 14. Percepción de autoeficacia en habilidades de análisis (HA) según aspectos socio-demográficos y de trayectoria académica

Fuente: Los autores

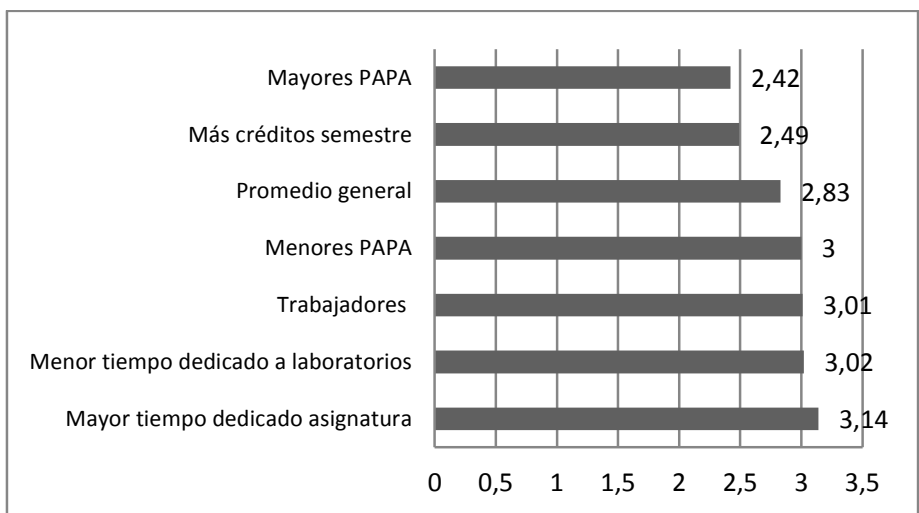

Figura 15. Percepción de utilidad como estrategia didáctica según aspectos socio demográficos y de trayectoria académica Fuente: Los autores

En relación con el tipo de logro de aprendizaje que se fortaleció con los laboratorios virtuales implementados se encontró que según el promedio general no hay diferencia entre 


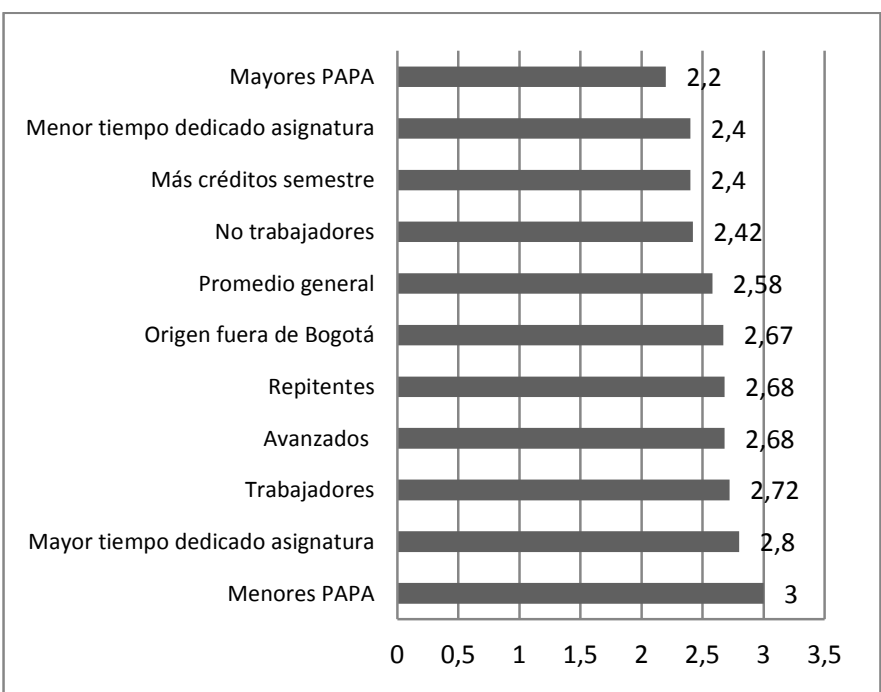

Figura 161. Evaluación de la experiencia según aspectos socio demográficos y de trayectoria académica

Fuente: Los autores

CC, HI y HA. En general, hubo un acuerdo ligeramente mayor hacia las habilidades de análisis (3) mientras que los otros dos son puntuados de igual manera (2,91). Profundizando en las respuestas, se encuentra que los estudiantes que dedicaron mayor tiempo a la asignatura y las mujeres perciben que este tipo de actividades favorece la comprensión de conceptos, con valoraciones de 3,67 y 3,5 respectivamente. Por otro lado, los estudiantes que reportaron mejores PAPA, los de menor edad y quienes no realizaron actividades laborales durante el semestre, presentaron más desacuerdo con este alcance de aprendizaje, con puntajes de 2,6; 2,6 y 2,4 respectivamente. Los grupos que presentaron mayor acuerdo con que los laboratorios virtuales permiten generar habilidades de análisis son las mujeres, los estudiantes que llevaban un porcentaje mayor del programa académico cursado, quienes pertenecían a programas de admisión especial y quienes reportaron mayor dedicación de tiempo a la asignatura con puntajes de 3,5; 3,4; 3,3 y 3,3 , respectivamente. Mientras que los grupos con mayor desacuerdo con este alcance de aprendizaje fueron los que han cursado menor porcentaje del programa académico y aquellos que matricularon mayor número de créditos durante el semestre, con puntajes de 2,67 y 2,5, respectivamente. Finalmente, en relación con las HI fueron las mujeres y los estudiantes con menores PAPA quienes presentaron mayor acuerdo con que estas son fortalecidas con los laboratorios virtuales, con puntajes de 3,5 y 3,3 respectivamente. Los grupos de mayor desacuerdo frente a este logro de aprendizaje fueron los estudiantes con mejores PAPA, quienes cursaron menos créditos en el semestre y quienes reportaron una menor dedicación de tiempo a la asignatura durante el semestre, con puntajes de 2,6; 2,5 y 2,5 respectivamente.

\subsection{Evaluación de la experiencia asociada con el tipo de estudiante}

Las afirmaciones de esta categoría fueron evaluadas según la misma escala Likert de 4 opciones. Separando en dos categorías el grupo de preguntas, se tienen aquellas relacionadas con el acompañamiento y las que evaluaban de manera general la experiencia. En este último grupo, el promedio de evaluación general fue de 2,58.

Los mejores promedios de evaluación de la experiencia (Fig. 16) corresponden a los grupos de estudiantes con menor PAPA (3), con mayor tiempo de dedicación a la asignatura $(2,8)$ y quienes trabajaron en el semestre $(2,72)$. De otro lado, las peores evaluaciones corresponden a los estudiantes con mayor PAPA $(2,2)$, quienes cursaron más créditos en el semestre $(2,4)$, dedicaron menos tiempo a la asignatura $(2,4)$ y no trabajaron durante el semestre $(2,42)$. Al indagar sobre la afirmación "No necesito acompañamiento para resolver este tipo de actividades (docentes, monitores, recurrir a otras personas)” la mayoría estudiantes agrupados por características socio-demográficas y trayectoria académica presenta desacuerdo, con puntajes de 2 o menos, con excepción de quienes dedicaron menos tiempo a la asignatura $(2,7)$, quienes reportaron provenir de fuera de Bogotá $(2,6)$, los de mayor edad $(2,2)$, quienes trabajaron en el semestre $(2,1)$ y quienes repetían la asignatura $(2,1)$. La mayoría puntúa más de 2,5 en relación con el uso de recursos y la búsqueda de apoyo cuando requirió ayuda, excepto quienes dedicaron menos tiempo a la asignatura que puntuaron en promedio 2,2 en cuanto a la búsqueda de ayuda cuando se requiere, quienes trabajaron $(2,3)$ y quienes llevan mayor parte del programa académico cursado $(1,8)$.

\section{Conclusiones}

Los laboratorios virtuales son una herramienta que permite a los estudiantes potenciar sus habilidades de análisis y de investigación en la solución problemas específicos de la ingeniería. Así mismo se perciben como pertinentes para permitir a los estudiantes aproximarse a lo que será su rol como ingenieros en contextos laborales. Pero para alcanzar estos propósitos es fundamental que los laboratorios, virtuales o presenciales, estén bien fundamentados y diseñados [34]. En el caso del fortalecimiento de las habilidades de resolución de problemas debe atenderse principalmente su estructura que en este caso implicó la construcción de los problemas y las preguntas alrededor de estos. A partir del diseño propuesto, se logró la movilización de algunos elementos del sistema de aprendizaje [27] en el que estaban inmersos los estudiantes. Sin embargo, los resultados de las percepciones de los estudiantes permiten sugerir la necesidad de movilizar otros elementos, dentro de los que se encuentran los agentes educativos que realizan el acompañamiento en la realización de los laboratorios, así como la articulación entre la asignatura y los laboratorios virtuales, transformando otras de sus estrategias además de los laboratorios.

Los estudiantes percibieron los laboratorios virtuales como más complejos de realizar que otros que han realizado. Esto se debe principalmente a dos asuntos: el primero la recepción de una amplia cantidad de datos; y el segundo, que el tipo de preguntas a resolver no están centradas en procedimientos. Lo anterior puede relacionarse con la respuesta que dieron en el cuestionario indicando que no quieren más actividades como esta en sus asignaturas. Sin embargo, con la indagación en el grupo focal, se encontró que esta experiencia permitió transformar las relaciones estudiantes-datos y datos-problemas; 
propició que los estudiantes se exigieran a analizar e interpretar el problema y sus preguntas; a probar diferentes formas de abordaje; y, a elegir entre las diversas rutas para abordar el problema que los llevaban a resultados distintos [20], [28]. Si bien la complejidad percibida les resultó en principio negativa, los estudiantes valoraron positivamente el reto que esto supuso para su formación y para la construcción de su identidad como ingenieros en formación.

De otro lado, se considera importante seguir profundizando sobre el desarrollo de habilidades instrumentales de los estudiantes. Los laboratorios virtuales no tienen como propósito el desarrollo de dichas habilidades en tanto tienen como supuesto fundamental que no haya una relación directa de los estudiantes con los instrumentos de laboratorio. Sin embargo, de acuerdo con lo manifestado por los estudiantes, en los espacios presenciales hay debilidades para el desarrollo de estas habilidades. Por lo tanto, el diseño de nuevas experiencias con laboratorios virtuales podría buscar opciones para articular estratégicamente en el sistema de aprendizaje el desarrollo de habilidades instrumentales.

Tanto los resultados de autoeficacia como de percepción varían al analizarlos según las características sociodemográficas de los estudiantes. De esta manera, resulta pertinente a futuro explorar diseños didácticos que incorporen laboratorios virtuales cuya flexibilidad permita adaptaciones concretas a los intereses y características de los grupos que componen cada curso. También se encuentran diferencias según la trayectoria académica de los estudiantes y el tiempo de estudio dedicado a la asignatura. Los estudiantes que más tiempo dedicaron a ésta valoran de manera más positiva el trabajo realizado con estos laboratorios virtuales y su capacidad de aportar en el desarrollo de conceptos y habilidades de análisis.

La ejecución de laboratorios virtuales se evidencia como una estrategia pertinente para dinamizar el pensamiento hacia la resolución de problemas, en tanto moviliza conocimientos, habilidades de investigación y de análisis. Sin embargo, para afianzar su desarrollo se requiere un mayor ejercicio de acompañamiento y retroalimentación. Al respecto, es clave que quienes ofrezcan la retroalimentación no solo tengan conocimientos técnicos sobre los ensayos, sino que comprendan la óptica del ABP y brinden orientaciones técnicas generales, respondan preguntas concretas sobre los datos y fortalezcan el pensamiento orientado a la resolución de problemas.

\section{Referencias}

[1] Kirsh, D., Problem solving and situated cognition, de The Cambridge handbook of the learning sciences, 2009.

[2] Colmenares, J.E. y Celis, J., Hacia una formación más fundamentada y flexible en ingeniería civil, Revista Educación en Ingeniería, 11(21), pp. 4-8, 2016. DOI: 10.26507/rei.v11n21.581

[3] Holtz, R.D. y Kovacs, W., An introduction to geotechnical engineering, Prentice Hall, 1981.

[4] Mitchell, J.K. and Soga, K., Fundamental of soils behavior, 3rd Edition. ed., John Wiley \& Sons, 2005.

[5] Knappett, T.W. and Whitman, R.V., Craig's soil mechanics, 8th Edition. ed., Spon Press, 2012.

[6] Das, B.M. and Sobhan, K., Principles of geotechnical engineering, 8th Edition. ed., Cengage Learning Inc., 2013.
[7] Powrie, W., Soil mechanics: Concepts and applications, 3rd Edition. ed., CRC Press, 2014.

[8] Budhu, M., Soil mechanics fundamentals, Wiley-Blackwell, 2015.

[9] Prince, M.J. and Felder, R.M., Inductive teaching and learning methods: Definitions, comparisons, and research bases, Journal of Engineering Education, 95(2), pp. 123-138, 2006. DOI: 10.1002/j.21689830.2006.tb00884.x

[10] McDowell, G., A student-centred learning approach to teaching soil mechanics, International Journal of Engineering Education, 17(3), pp. 255-260, 2011.

[11] Brinson, J., Learning outcome achievement in non-traditional (virtual and remot) versus traditional (hands on) laboratories, Computers and Education, 87, pp. 218-237, 2015. DOI: 10.1016/j.compedu.2015.07.003

[12] de Jong, T., Linn M. and Zacharia, Z., Physical and virtual laboratories in science and engineering education, Science, 340, pp. 305-308, 2013. DOI: 10.1126/science.1230579

[13] Stefanovic, M., Tadic, D. and Nestic, S., An assesment of distance learning laboratory objectives for control engineering education, Computer application in engineering education, 23(2), pp. 191-202, 2013. DOI: $10.1002 /$ cae.21589

[14] Wolf, T., Assessing student learning in a virtual laboratory environment, IEEE Transactions on Education, 53(2), pp. 216-222, 2010. DOI: 10.1109/TE.2008.2012114

[15] Haque, M.E., Interactive animation and visualization in a virtual soil mechanics laboratory, de Frontiers in Education Conf. 31st Annu, Reno, 2001. DOI: 10.1109/FIE.2001.963840

[16] Penumadu, D. and Prashant, A., Virtual simulator for advances geotechnical laboratory testing, in: Conf. Geo Congress, Atlanta, 2006. DOI: $10.1061 / 40803(187) 270$

[17] Maurel, M.d.C., Dalfaro, N.A. y Soria, H.F., El laboratorio virtual: Una herramienta para afrontar el desgranamiento, en: Congreso Iberoamericano de Ciencia, Tecnología, Innovación y Educación, Buenos Aires, 2014.

[18] Universidad Nacional de Colombia, Documento interno de trabajo. Programa de ingeniería civil., 2016.

[19] Consejo de la Facultad de Ingeniería Sede Bogotá, Acuerdo 27 de 2015, de 29 de octubre de 2015. [en línea]. Disponible en: http://www.legal.unal.edu.co/sisjurun/normas/Norma1.jsp?i=85846

[20] Colmenares, J.E., Héndez-Puerto, N.R. y Celis-Giraldo, J.E., Laboratorios virtuales desde la perspectiva de resolución de problemas: Caso de la asignatura de mecánica de suelos, Revista Educación en Ingeniería, 12(22), pp. 97-103, Julio 2016. DOI: 10.26507/rei.v11n22.705

[21] Universidad Nacional de Colombia, Informe de autoevaluación. Renovación de acreditación. Programa de pregrado en ingeniería civil., Bogotá, 2010.

[22] Arévalo, C.E. y Bulla, L.A., Laboratorios virtuales para el aprendizaje en ingeniería civil a distancia, Revista Academia y Virtualidad, 1(1), pp. 7381, 2008.

[23] Celis, J., Camacho, A., León-Arenas, A. y Duque, M., Dime cómo enseñas y te diré qué tanto aprenden los estudiantes. Los aprendizajes y las prácticas de aula en algunas facultades de ingeniería en Colombia, Bogotá: ACOFI, ICFES, UniAndes, UniNorte, 2014.

[24] Colmenares, J., Syllabus de la asignatura mecánica de suelos, 2015.

[25] Díaz-Barriga, F., Cognición situada y estrategias para el aprendizaje significativo, Revista Electrónica de Investigación Educativa, 5(2), pp. 1-12, 2003.

[26] Greeno, J.G., Learning in activity, in: The Cambridge Handbook of the Learning Sciences, Sawyer, K., Ed., St. Louis, Washington University, 2006, pp. 79-96.

[27] Johri, A. and Olds, B., Situated engineering learning: Bridging engineering education research and the learning sciences, Journal of Engineering Education, 100(1), pp. 151-185, 2011. DOI: 10.1002/j.2168-9830.2011.tb00007.x

[28] Zareba, M., Schuh, A. and Camelio, J., Accelerated Problem solving sessions in university laboratory settings, Journal of Intelligent Manufacuring, 24(3), pp. 517-526, 2013. DOI 10.1007/s10845-0110558-9 
[29] Mitchell, J.E., Canavan, B. and Smith, J., Problem-based learning in communication systems: Student perceptions and achievement, IEEE Transactions on Education, 53(4), pp. 587-594, 2010. DOI: 10.1109/TE.2009.2036158

[30] Flores, J.,M. Caballero, C. y Moreira, M.A., El laboratorio en la enseñanza de las ciencias: Una visión integral en este complejo ambiente de aprendizaje, Revista de Investigación, 33(58), pp. 75-111, 2009.

[31] Couto-Marques, J.M., Experimental, numerical and virtual tools in civil engineering, de Global Engineering Education Conference (EDUCON), Amman, 2011. DOI: 10.1109/EDUCON.2011.5773293

[32] Gibbins, L. and Perkin, G., Laboratories for the 21st Century in STEM Higher Education, Loughborough: Loughborough University, 2013.

[33] Rosado, L. y Herreros, J.R., Aportaciones didácticas de los laboratorios virtuales y remotos en la enseñanza de la física, de Congreso TAEE Tecnología Aprendizaje y Enseñanza de la Electrónica, Valencia, 2004.

[34] White, R., The link between the laboratory and learning, International Journal of Science Education, 18(7), pp. 761-774, 1996. DOI: $10.1080 / 0950069960180703$

[35] Lévy, P., Cibercultura. La cultura de la sociedad digital, Barcelona: Antrophos, 2007.

[36] Scolari, C., Interfaces para saber, interfaces para hacer. Las simulaciones digitales y las nuevas formas del conocimiento, en: Educomunicación: más allá del 2.0, Barcelona, Gedisa, 2010, pp. 225-250.

[37] Chamizo, J.A. e Izquierdo, M., Evaluación de las competencias de pensamiento científico, Alambique. Didáctica de las ciencias experimentales, 51, pp. 9-19, 2007.

[38] Ávila-Fajardo, G.P. y Riascos-Erazo, S.C., Propuesta para la medición del impacto de las TIC, Revista Educación y Educadores, 14(1), pp. 169188, 2011.

[39] Leal, D., Evaluación de aprendizaje en entornos en línea, abiertos y distribuidos, en: La educación superior a distancia y virtual en Colombia: Nuevas realidades, Arboleda-Toro, N. y Rama-Vitale, C., Eds., Bogotá, Virtual Educa; ACESAD, 2013, pp. 155-174.

[40] Álvarez-Álvarez, C. y San Fabián-Maroto, J.L., La elección del estudio de caso en investigación educativa, Gazeta de Antropología, 28(1), pp. 1-12, 2012.

[41] Aktam-Takin, N. and Vatansever, F., A Web-based virtual power electronics laboratory., Computer Application in Engineering Education, 24, pp. 71-78, 2016. DOI: 10.1002/cae.21673

[42] Bandura, A., Guide for constructing self-efficacy scales, in: Self efficacy beliefs of adolescents, Colorado, University of Colorado School University, 2006, pp. 307-337.

[43] Bryman, A., Social research methods, Oxford: Oxford University Press, 2008.

[44] SNIES, Matriculados en educación superior 2015, 2015.
J.E. Colmenares, es Ing. Civil en 1989 y MSc. en Geotecnia en 1996, de la Universidad Nacional de Colombia, Bogotá; MSc. y DIC en1997 y Dr. of Philosophy en 2002 del Imperial College de la Universidad de Londres, Reino Unido. Es profesor titular de la Facultad de Ingeniería de la Universidad Nacional de Colombia, Bogotá, Colombia, en donde desarrolla actividades académicas desde 1992. Sus intereses de investigación están concentrados en el estudio experimental del comportamiento mecánico de los suelos, la mecánica de suelos no-saturados y en la enseñanza de la Ingeniería.

ORCID: 0000-0002-1485-0327

N.R. Héndez, es Psicóloga en 2004 de la Universidad Santo Tomás, Bogotá; Esp. en Desarrollo Humano con Énfasis en Procesos Afectivos y Creatividad en 2007 de la Universidad Distrital “Francisco José de Caldas”, Bogotá; y MSc en Investigación en Didáctica, Formación y Evaluación Educativa en la Universidad de Barcelona, Barcelona. Es miembro de SOLACyT Capítulo Colombia. Ha sido asesora pedagógica de diferentes proyectos de educación superior y extensión en la Universidad Nacional de Colombia, Universidad Católica y Universidad Central, Bogotá, Colombia. Sus intereses investigativos incluyen: incorporación de TIC para la enseñanza y el aprendizaje; formación científica; formación docente, educación superior.

ORCID: 0000-0002-5339-4408

J. Celis, recibió el título de Sociólogo en 2001, el título de MSc. en Sociología en 2003 de la Universidad Nacional de Colombia, Bogotá, Colombia y el título de MSc. en Estudios Comparados e Internacionales en Educación en 2015 de la Universidad de Estocolmo, Estocolmo, Suecia. Ha trabajado como consultor para COLCIENCIAS, Ministerio de Educación Nacional, Banco Mundial, entre otros. Ha sido investigador en las Universidades Nacional de Colombia y Andes. Sus intereses investigativos incluyen: formación doctoral; inserción de doctores al sector productivo; transferencia tecnológica; educación media. ORCID: 0000-0003-0410-5953 